\title{
A priori estimation of sequencing effort in complex microbial metatranscriptomes
}

\author{
Toni Monleon-Getino ${ }^{1,2}$ (D) | Jorge Frias-Lopez ${ }^{3}$
}

\author{
${ }^{1}$ Section of Statistics (Department of \\ Genetics, Microbiology, and Statistics), \\ University of Barcelona, Barcelona, Spain \\ ${ }^{2} \mathrm{BIOST}^{3}$, GRBIO (Research Group in \\ Biostatistics and Bioinformatics), Barcelona, \\ Spain \\ ${ }^{3}$ College of Dentistry, University of Florida, \\ Gainesville, FL, USA
}

\section{Correspondence}

Toni Monleon-Getino, Section of Statistics (Department of Genetics, Microbiology, and Statistics), Faculty of Biology, Avda Digonal 643, 08028 Barcelona, Spain.

Email: amonleong@ub.edu

\begin{abstract}
Metatranscriptome analysis or the analysis of the expression profiles of whole microbial communities has the additional challenge of dealing with a complex system with dozens of different organisms expressing genes simultaneously. An underlying issue for virtually all metatranscriptomic sequencing experiments is how to allocate the limited sequencing budget while guaranteeing that the libraries have sufficient depth to cover the breadth of expression of the community. Estimating the required sequencing depth to effectively sample the target metatranscriptome using RNAseq is an essential first step to obtain robust results in subsequent analysis and to avoid overexpansion, once the information contained in the library reaches saturation. Here, we present a method to calculate the sequencing effort using a simulated series of metatranscriptomic/metagenomic matrices. This method is based on an extrapolation rarefaction curve using a Weibull growth model to estimate the maximum number of observed genes as a function of sequencing depth. This approach allowed us to compute the effort at different confidence intervals and to obtain an approximate a priori effort based on an initial fraction of sequences. The analytical pipeline presented here may be successfully used for the in-depth and time-effective characterization of complex microbial communities, representing a useful tool for the microbiome research community.
\end{abstract}

\section{KEYWORDS}

machine learning, metagenomics, metatranscriptomics, NGS, rarefaction curve, sample size, sequencing effort, simulation
The study of the human microbiome has dramatically expanded our understanding of the role that microbes play in health and disease. These studies have been facilitated by the development of next-generation sequencing (NGS) technologies, which are capable of generating enough sequences to cover most of the diversity

\section{1 | INTRODUCTION}

This is an open access article under the terms of the Creative Commons Attribution License, which permits use, distribution and reproduction in any medium, provided the original work is properly cited.

(c) 2020 The Authors. Ecology and Evolution published by John Wiley \& Sons Ltd. present in a sample. However, capturing the full composition is still a challenge, even when estimated by the distribution of 16S rDNA sequences ( $\mathrm{Ni}$ et al., 2013; Tamames et al., 2012). The study of transcriptomes of whole microbial communities, or metatranscriptomics, has increased exponentially in the last few years (Shakya et al., 2019; Zhang et al., 2019), and trying to analyze these kind of data has produced a new set of challenges. Thus, García-Ortega 
and Martínez (2015), using a nonparametric estimator for the number of undetected genes, found that on average, approximately $10 \%$ of the expressed genes per accession remain undetected if individual sequencing libraries are analyzed. The power and accuracy of such experiments depend substantially on the number of reads sequenced, so a crucial step in the experiment design should be determining the optimal read depth for a particular study or verifying whether the experiment has adequate depth (Robinson \& Storey, 2014).

Most RNA-seq studies have focused on assessing the depth of transcriptome sequencing in eukaryotic systems, using a wide range of estimated sequencing depths to cover the full patterns of expression. In the human transcriptome, the sequencing depth estimated as necessary to observe differences in expression profiles varies from 100 to 700 million sequences (Toung et al., 2011; Westermann et al., 2012). In the case of prokaryotic RNA-seq experiments, Haas et al. have shown that the reads typically produced in a single lane of the Illumina HiSeq sequencer far exceed the number needed to saturate the annotated transcriptomes of diverse bacteria growing in monoculture (Haas et al., 2012).

In metatranscriptome sequencing, saturation is reached when an increment in the number of reads does not result in an additional increment in the number of expressed transcripts, or no additional ORFs are detected in the case of shotgun metagenomic analysis. One way of estimating the point of saturation is by using rarefaction curves, a method commonly used in ecology to estimate the species richness as a function of sampling effort. In the case of RNA-seq/ DNA-seq, a higher sequencing depth will only prolong the curve but is otherwise comparable to a lower sequencing depth curve for the same regions. Once the curve reaches a plateau, where additional sequencing would only marginally increase the number of transcripts observed, the curve can be considered as saturated, and there is therefore no need to increase the sequencing effort to describe the gene expression profiles of the community. Another useful feature of saturation curves is that they allow the complexity of the sample to be assessed: Expressed transcripts will be numerous in highly complex communities and low in those with low complexity.

We have developed a method to calculate the sequencing effort needed to reach the maximum number of existing genes using rarefaction curves extrapolating from a small initial sequencing depth (10\%-20\%) and estimating the confidence intervals at $90 \%, 95 \%$, or $99 \%$ of the maximum sequencing effort.

\section{2 | MATERIAL AND METHODS}

\section{1 | Methodological overview}

We first simulated more than a thousand different metatranscriptomic/metagenomic matrices. On those matrices, we computed rarefaction curves using the function iNEXT( ) from the iNEXT R library for Interpolation and Extrapolation for Species Diversity (Hsieh et al., 2016). We then used a nonlinear growth model to compute the maximum number of genes expected and to estimate the sequencing depth (reads) required for $90 \%, 95 \%$ or $99 \%$ of the maximum sampling effort.

Finally, using a method based on machine learning, we predicted the $90 \%, 95 \%$, or $99 \%$ of the maximum number of genes using only a minimum number of sequencing depth (reads) and the sampling effort needed. All these functionalities were included in some functions of R. The method was tested, as an application thereof, on metatranscriptomic samples of an oral microbiome. The results are presented in the supplementary material of this article.

\section{2 | Simulation of metatranscriptomic/ metagenomic matrices}

Metatranscriptomic/metagenomics matrices were simulated as described in Rodríguez-Casado et al. (2017) and in Monleon-Getino et al. (2019). In order to simulate gene data and the associated reads in each sample, it is necessary to know which underlying probability model best explains the distribution of the data, for example, the binomial (distribution of the reads per gene in a sample), multinomial (distribution of the counts for the set of genes in the sample), and complex distributions such as the Dirichlet-multinomial (distribution of reads for the set of genes and samples in the experiment). The following is a brief theoretical introduction to these distributions, which once known allow the simulation of new samples by MonteCarlo simulation, a statistical method used to solve complex mathematical problems through the generation of random variables.

Table 1 shows the general metatranscriptomic/metagenomic matrix $(M)$ structure ( $n$ rows: samples, $p$ columns: genes) obtained after the bioinformatic analysis, which constitutes the starting point of this study. Outlined below are the mathematical formalization and the study of probability distribution, previously studied in more depth (Monleon-Getino et al., 2019).

\begin{tabular}{lllllll} 
Number & Gene & Sample 1 & Sample 2 & Sample jth & Sample $n$ & Total \\
\hline 1 & type. 1 & $m_{11}$ & $m_{12}$ & $\ldots$ & $m_{1 n}$ & $N_{1 .}$ \\
\hline 2 & type. 2 & $m_{21}$ & $m_{22}$ & $\ldots$ & $m_{2 n}$ & $N_{2 .}$ \\
$\vdots$ & $\vdots$ & $\ldots$ & $\ldots$ & $m_{i j}$ & $\ldots$ & $\ldots$ \\
\hline$k$ & type. $k$ & $m_{k 1}$ & $m_{k 2}$ & $\ldots$ & $m_{k n}$ & $N_{k .}$ \\
\hline & Total & $N_{.1}$ & $N_{.2}$ & $\ldots$ & $N_{. n}$ & $N$ \\
\hline
\end{tabular}

TABLE 1 Data matrix structure of $\mathbf{M}^{\prime}$ (metatranscriptomics or metagenomics matrix input) 
Usually, for convenience, in $M$ we change the notation of $p$ by $k$; also during the statistical analysis, we use the transpose $\mathbf{M}^{\prime}$ structure ( $k$ rows: genes, $p$ columns: samples), which shows the samples (e.g. individuals) in the columns and the identified gene in the rows (Table 1).

As a result of genomic analysis, $\boldsymbol{M}^{\prime}$ can be very large and usually has thousands of genes, most of them with small frequencies or 0 , that is, $M^{\prime}$ is typically a sparse matrix. This matrix is truncated because some characteristics were not observed in the sampling.

From the statistical point of view, it is highly convenient to formalize the probability distribution underlying this matrix structure, so each sample from $M^{\prime}$ can be represented by one $k$-dimensional random vector $X_{j} ; X_{j}=\left(m_{1 j}, m_{2 j}, \ldots, m_{k j}\right)$, where $m_{k j}$ represents the number of times that gene $k$ is observed in sample $j$.

The probability distribution of each random vector $X_{i}$. (vector row) and $X_{j}$ (vector column) can be associated individually with a multinomial distribution,

$$
\begin{aligned}
& X_{. j} \sim M N\left(N_{. j}, \theta_{1 j}, \ldots, \theta_{k j}\right) ; \forall j=1, \ldots, n \\
& X_{i .} \sim M N\left(i ., \theta_{i 1}, \ldots, \theta_{i n}\right) ; \forall i=1, \ldots, k
\end{aligned}
$$

The multinomial distribution is a multivariate generalization of the binomial distribution, where the marginal distribution of each $X_{i j}$ is:

$$
X_{i j} \sim \operatorname{Bin}\left(m_{i j}, \theta_{i j}\right) ; 1 \leq \theta_{i j} \leq 1 ; \forall j=1, \ldots, n ; \forall i=1, \ldots, k
$$

for example, if we consider the partition of all sample space $\Omega^{j}$ the $j$-sample space in $k$ parts:

$$
A_{1 j}, A_{2 j}, \ldots, A_{k j}
$$

One individual selected randomly has the probability $\theta_{k j}$ of belonging to the gene $A_{k j}$ in the partition:

$$
\left.\begin{array}{c}
P\left(A_{1 j}\right)=\theta_{1 j} \\
P\left(A_{2 j}\right)=\theta_{2 j} \\
\vdots \\
P\left(A_{k j}\right)=\theta_{k j}
\end{array}\right\} \sum_{i=1}^{k} \theta_{i j}=1 ; \forall j=1, \ldots, n
$$

If we wish to calculate the probability of sample $j$ having $N_{j}$ individuals, $m_{1 j}$ belongs to class $A_{1 j}, m_{2 j}$ to class $A_{2 j}, \ldots, m_{k j}$ to class $A_{k j}$, with the restriction

$$
\sum_{i=1}^{k} m_{i j}=N_{. j} ; \forall j=1, \ldots, n
$$

Furthermore, using the multinomial function of density (mass function) we can calculate this probability, $\operatorname{MN}\left(N_{j} ; \theta_{j}=\left(\theta_{1 j}, \theta_{2 j}, \ldots, \theta_{k j}\right)\right)$
$P\left[\left(A_{1 j}=m_{1 j}\right) \cap \ldots \cap\left(A_{h j}=n_{k j}\right)\right]=\frac{N_{j} !}{m_{1 j} ! m_{2 j} ! \ldots m_{k j} !} \theta_{1 j}^{m_{1 j}} \cdot \theta_{2 j}^{m_{2 j}} \cdot \ldots \cdot \theta_{k j}^{m_{k j}} ; \forall j(6)$

where $0 \leq \theta_{i j} \leq 1$ for all $i$ in 1 to $k$, and $\theta_{1 j}+\ldots+\theta_{k j}=1(\forall j)$, and if $k=1$, the mass function is reduced to the binomial, $\forall j=1, \ldots, n$.

The conjugate prior of the Multinomial distribution is the Dirichlet distribution, the multivariate generalization of beta distribution. Hence, the parameter vector $\theta_{k}=\left(\theta_{1 j}, \theta_{2 j}, \ldots, \theta_{k j}\right) ; \forall j$ has a prior distribution given by:

$$
\theta_{k} \sim \operatorname{Dirichlet}\left(\alpha_{1 j}, \alpha_{2 j}, \ldots, \alpha_{k j}\right) ; \forall j=1, \ldots, n
$$

In (10), the density function is given by:

$$
\begin{gathered}
g\left(\theta \mid \alpha_{1 j}, \alpha_{2 j}, \ldots, \alpha_{k j}\right)=\frac{\Gamma\left(\sum_{i}^{k} \alpha_{i j}\right)}{\prod_{i}^{k}\left(\Gamma \alpha_{i j}\right)} \theta_{1 j}^{\left(\alpha_{1 j}-1\right)} \theta_{2 j}^{\left(\alpha_{2 j}-1\right)} \ldots \theta_{k j}^{\left(\alpha_{k j}-1\right)} ; \\
\alpha_{i j}>0 ; 0 \leq \theta_{i j} \leq 1 ; \sum_{i}^{k} \theta_{i j}=1 ; \forall j=1, \ldots, n
\end{gathered}
$$

In Bayesian inference, $p(\theta \mid x)$ is known as posterior distribution and is proportional to likelihood $(p(x \mid \theta)) x$ prior distribution $(p(x))$, so $p(\theta \mid x) \propto p(x \mid \theta) \cdot p(x)$.

The posterior distribution of $\theta_{j}$ given $X$ is:

$$
\theta_{j} \mid x \sim \operatorname{Dirichlet}\left(x_{1 j}+\alpha_{1 j}, x_{2 j}+\alpha_{2 j}, \ldots, x_{k j}+\alpha_{k j}\right) ; \forall j=1, \ldots, n
$$

Thus, in order to implement a new method that calculates the depth of the sample and conveniently estimates the sampling effort, as well as whether it is necessary to sequence more samples or not, matrices $M^{\prime}$ can be simulated with different values of $k$ and $n$, with $M^{\prime}$ requiring a multinomial probability distribution. $\mathbf{M}^{\prime}$ can be directly simulated from the joint posterior Dirichlet distribution, using the rdirichlet( ) function from the LearnBayes package in R (CRAN, 2018a, 2018b) and the rmultinom( ) function with Dirichlet prior probability (Monleon-Getino et al., 2019).

\section{3 | Calculating rarefaction curves}

There are many methods for calculating the rarefaction curve for each $M^{\prime}$; here, we chose to use one of the most recent ones, the iNEXT( ) function of R iNEXT for Interpolation and Extrapolation for Species Diversity (Hsieh et al., 2016). This library provides simple functions to compute and plot two types (sample size- and coverage-based) of rarefaction and extrapolation of species diversity (based on Hill numbers) for individual-based (abundance) data or sampling unit-based (incidence) data. 
Using the iNEXT( ) function, we calculated the rarefaction curves for each metatranscriptomic/metagenomic matrix $\left(M^{\prime}\right)$ simulated previously.

\subsection{Calculating sampling effort}

Unfortunately, iNEXT( ) cannot calculate the maximum number of genes or estimate the sampling effort, and the reads covering $90 \%$, $95 \%$, and $99 \%$ of the maximum number of genes in the case of nonsaturated rarefaction curves. To address this caveat, we propose a nonlinear parametric model.

In this type of study, it is common to perform an initial analysis for model selection. Thus, rarefaction curves were first fitted based on previous experience and a selection of possible nonlinear models (Mendez et al., 2017) or the use of Bayesian methods (MonleonGetino et al., 2017) were tested.

Several functions, including Weibull, logistic, asymptotic regression through the origin (or a two-parameter Weibull growth model), Gompertz, and Michaelis-Menten models, were tested using nonlinear regression for use as extrapolations of the rarefaction curves (Mendez et al., 2017). The regression analysis was performed using the R-package function nls( ), and the model accuracy was tested with the function accuracy( ) of the R-package rcompanion (R Companion, 2018), which produces a table of statistics that can fit multiple models. The model accuracy was tested using Efron's pseudo r-squared, Min.max.accuracy (for minimum, maximum accuracy, more substantial indicates a better fit, and a perfect fit is equal to 1 ), and root-mean-square error (RMSE), which has the same units as the predicted values. The Weibull sigmoid model obtained the best scores and was selected as a useful function that fits and extrapolates the rarefaction curve.

The Weibull growth model used in our studies is derived from the one-parameter Weibull function (10), given by:

$$
F(x)=1-e^{\left(-x^{\prime}\right)}
$$

where $\gamma$ is a shape parameter and $x>0$ and $\gamma>0$. The distribution function has a point of inflection at $(x, F(x))=\left(\frac{[(\gamma-1) / \gamma]^{1}}{\gamma}, 1-\exp \left(-\left(1-\gamma^{-1}\right)\right)\right)$. The following equation can then be used to obtain the sigmoidal curve for empirical use:

$$
F(x)=\beta+(\alpha-\beta) F(k x, \theta)
$$

Moreover, the Weibull function of four parameters can be described by the function $F(x)=\alpha-(\alpha-\beta) e^{-(k x)^{\gamma}}$. Thus, in our case the Weibull growth model of four parameters (Pinheiro, 2018) is described by the function $W(x)$ :

$$
W(x)=a-b e^{-(c x)^{m}}
$$

where $W(x)$ is the potential number of genes being expressed for each number of reads $(x)$ and now $a=\alpha, b=\alpha-\beta, c=\kappa^{\gamma}$ and $m=\gamma \cdot a, b, c$, and $m$ are parameters to be estimated and $e$ is the base of the natural logarithms. $a$ is the asymptote of limiting value of the response variable $W(x), x \infty$ lim $W(x)=a$, which represents the maximum number of expressed genes. $b$ is the biological constant (lower asymptote), $c$ is the parameter governing the rate at which the response variable approaches its potential maximum $a$, and finally, $m$ is the allometric constant. The four-parameter Weibull growth model is considered very flexible in that it can be easily transformed into a three-, two-, or one-parameter model to adapt the relation between possible numbers of genes being expressed for each sample size (reads). For example, by setting $b=a$ and $m=1$ from (12), we obtained a two-parameter Weibull growth model (or Asymptotic regression through the origin model given by:

$$
W(x)=a\left(1-e^{(-c x)}\right)
$$

with the same meaning $W(x), x, a$ and $c$ (see 12).

\subsection{Estimation of the amount of sequencing (reads) needed to cover the total expected microbial metatranscriptome/metagenome (confidence band)}

The maximum potential number of genes being expressed and the $95 \%$ confidence band was used as an estimation of the asymptote of limiting value in a Weibull growth model of four (12) or two parameters (13). Using this Weibull parametric model, we estimated the amount of sequencing needed to cover $90 \%, 95 \%$, and $99 \%$ of the total expected metagenome/metatranscriptome in the samples and the $95 \%$ confidence interval, based only on the first 1 million sequences for each sample. We used $R$ (v. 3.6) to perform all the calculations described below.

Parameters in the Weibull growth model were estimated using the nls (Nonlinear regression), nls2 (Nonlinear regression with brute force (CRAN, 2018b), and minpack.Im (R Interface to the Levenberg-Marquardt nonlinear least squares) packages. The option Ssweibull $(x ; a, b, c, m)$ was used for the four-parameter Weibull growth model, and $\sim \operatorname{SsasympOrig}(x ; a, b)$ was used for the two-parameter Weibull model. In order to initialize the parameters, a "bruteforce" algorithm was used, and then, the parameters were optimized until those that maximize the adjustment value were optimized; the "brute-force" algorithm returns the nls object corresponding to the starting values (CRAN, 2018b).

\subsection{A priori gene prediction using a few initial total reads}

We used different algorithms to fit a regression model to predict the potential number of genes, effort/reads covering $90 \%, 95 \%$, or $99 \%$ of the maximum number of genes based on the first $10 \%-20 \%$ of 
sequences (reads). As a first strategy, a classical linear regression of the function $\operatorname{Im}$ ( ) was optimized using a function step( ) to perform the stepwise model selection, and the model was validated using the function cv.Im (data, model, m) from the DAAG library (Maindonald \& Braun, 2010, 2019). This function gives internal and cross-validation measures of predictive accuracy for multiple linear regression.

Two other strategies were applied: the so-called machine learning algorithms such as support vector machines (SVM) and Extreme Gradient Boosting (XGBoost), in which we used the training data (with multiple features) $x_{i}$ (here the genes in each sequencing depth) to predict a target variable $y_{i}$ (maximum number of genes).

Support vector machines (SVM) constitute a data classification method that separates data using hyperplanes, which is useful in the case of regression (Cortes \& Vapnik, 1995). If we have labeled data, SVM can generate multiple separating hyperplanes, so that the data space is divided into segments, each containing only one kind of data. The SVM technique is generally useful for data which has nonregularity, that is, without a known distribution. We used the function SVM( ) in R for the calculation (Chang \& Lin, 2011).

Extreme Gradient Boosting is an efficient implementation of the gradient boosting framework from Chen and Guestrin (2016). Gradient boosting is a state-of-the-art prediction technique that sequentially produces a model in the form of linear combinations of simple predictors-typically decision trees-by solving an infinite-dimensional convex optimization problem. XBoost( ) from library Xboost ( ) in R (Chen \& Guestrin, 2016) permits the calculation of this predicted method.

In order to check the accuracy of different models, it is common to use the coefficient of determination ( $R^{2}$ or R-squared), the mean absolute error (MAE), and the root-mean-square error (RMSE) (Hyndman \& Koehler, 2006).

$R^{2}$ is the percentage of the response variable variation that is explained by the model:

$$
R^{2}=\text { Explained variation/Total variation }
$$

$R^{2}$ is always between 0 and 1,0 indicating that the model explains none of the variability of the response data around its mean and 1 that the model explains all the variability of the response data around its mean.

RMSE is a frequently used measure of the differences between values (sample and population values) predicted by a model or an estimator and the values observed. The RMSE represents the sample standard deviation of the differences between predicted and observed values.

$$
\mathrm{RMSE}=\frac{\sum_{i=1}^{n}\left(\hat{y}_{i}-y_{i}\right)^{2}}{n}
$$

where $n$ is the number of pairs of observations, $\widehat{y}_{i}$ the value predicted and $y_{i}$ the observed value.

Mean absolute error (MAE) is the average vertical distance between each point and the $Y=X$ line:

$$
M A E=\frac{\sum_{i=1}^{n}\left|\left(\hat{y}_{i}-y_{i}\right)\right|}{n}
$$

where $n$ is the number of pairs of observations, $\hat{y}_{i}$ the predicted value and $y_{i}$ the observed value.

\section{7 | Metatranscriptome databases used in the method application}

We used metatranscriptome datasets from three different sources for the application of the proposed method. The first set was generated in our lab as described in Yost et al. (2015) and is available at the Human Oral Microbiome Database (HOMD) server under the submission number 20141024 (ftp://ftp.homd. org/publication_data/20141024/RNA/ ). The second dataset was generated by Benítez-Páez et al. (2014) and is available at the MG-RAST server by accessing the "Oral Metatranscriptome" project, id 935 (http://metagenomics.anl.gov/linkin.cgi?proje $c t=935$ ). The third dataset was generated by Jorth et al. (2014) and is available at DNAnexus study number SRP033605 (http:// sra.dnanexus.com/studies/SRP033605). All databases were bioinformatically cleaned of rRNA sequences, and in the case of SRP033605, we also removed low-quality sequences from the query files. Fast clipper and fastq quality filters from the Fastx toolkit (http://hannonlab.cshl.edu/fastxtoolkit/) were used to remove sequences shorter than $50 \mathrm{bp}$ with a quality score $>20$ in $>80 \%$ of the sequence.

\section{3 | RESULTS AND DISCUSSION}

\section{1 | Metatranscriptomic/ Metagenomic matrix simulation, rarefaction computation, and estimation of parameters}

Our focus was to study the transcriptome of whole complex microbial communities rather than individual transcriptomes, using an oral community as a model. The oral microbiome is one of the best characterized human body sites (Belda-Ferre et al., 2012; Haffajee et al., 2008; Marsh, 2006; Paster et al., 2001; Peterson et al., 2013; Socransky et al., 1998), comprising an extremely complex and highly organized biofilm community (Kolenbrander, 2000; Kolenbrander et al., 2002). More than 700 bacterial species have been identified in the oral cavity [Paster et al., 2001; Dewhirst et al., 2010]. Many oral bacterial species have not yet been cultivated, and the only information we possess about them derives from their 16S rRNA phylogenetic affiliation.

In the current study, we investigated the proposed mathematical Weibull model, using nonlinear regression modeling. This model is a generalization of the asymptotic growth model in that it reduces when the parameter $m$ is unity (see Methods). 


\begin{tabular}{llll|}
\hline Estimations of parameters of interest & Mean & Minimum & Maximum \\
\hline Maximum number of genes observed & 183,312 & 6 & 926,470 \\
\hline $\begin{array}{l}\text { Effort computed using the } \\
\text { iNEXT( )+Weibull model }\end{array}$ & $72.399 \%$ & $1.208 \%$ & $100 \%$ \\
\hline $\begin{array}{l}\text { Reads covering } 99 \% \text { of the maximum } \\
\text { number of genes }\end{array}$ & $5,788,494$ & 80 & $31,779,350$ \\
\hline
\end{tabular}

TABLE 2 Estimations of parameters of interest using a set of 1,587 simulations by means of a multinomial model of a metatranscriptomic/ metagenomic matrix (a)

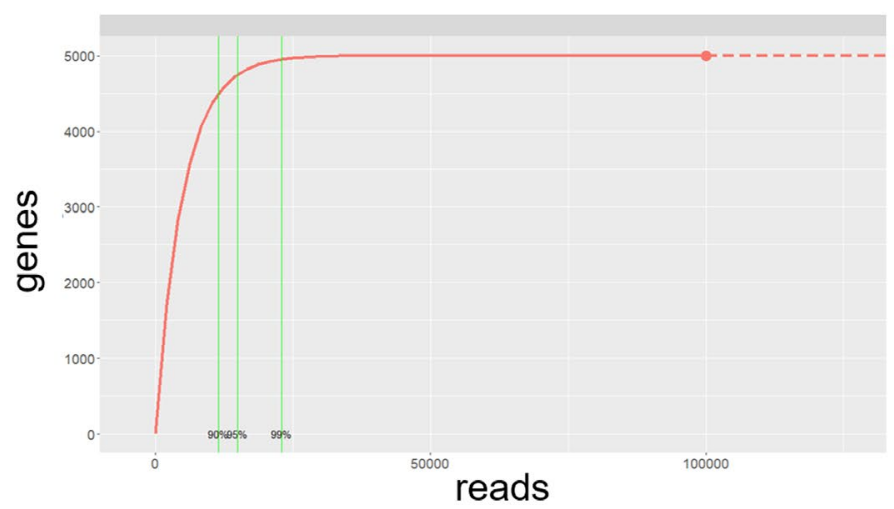

(c)

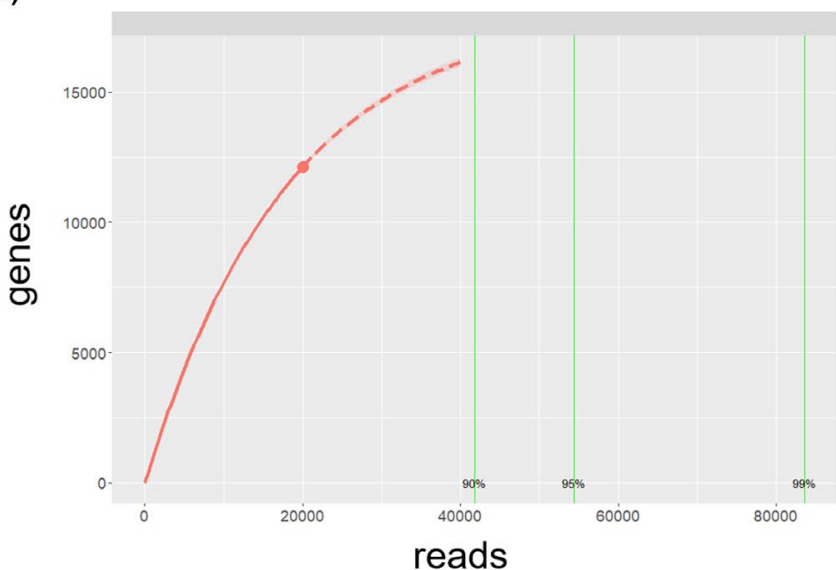

(b)

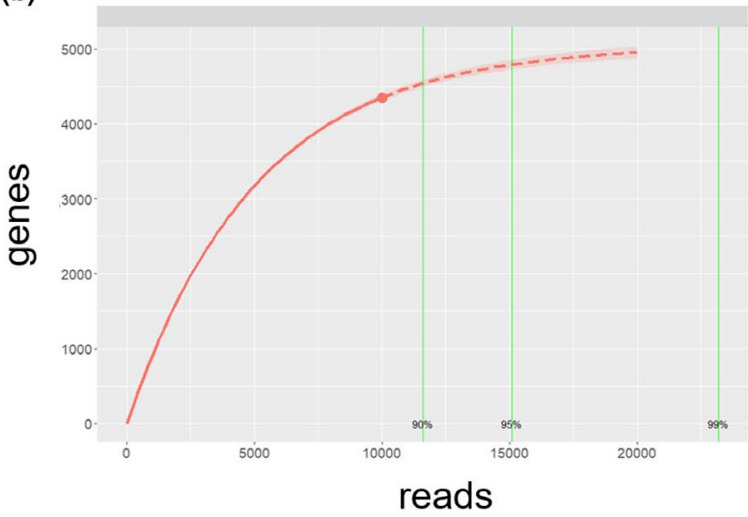

(d)

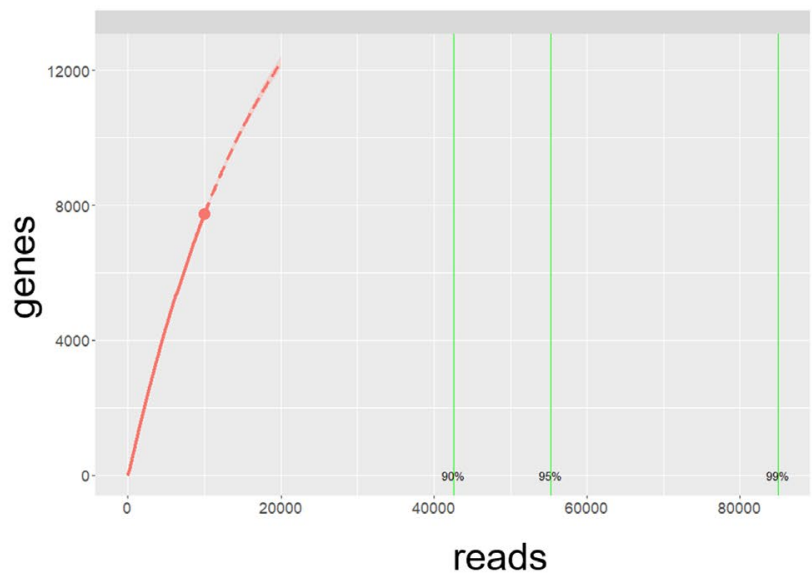

FIGURE 1 Calculation of the number of genes versus the number of reads using the PILI3() function of the library(Sequencingeffort). Interpolated data (solid red line), extrapolated data (dashed line), and red dot (limit of observed data)

\begin{tabular}{|c|c|c|}
\hline Model name & $\begin{array}{l}\text { Predictors used in the model (independent } \\
\text { variables, } X_{i} \text { ) }\end{array}$ & $\begin{array}{l}\text { Results }\left(R^{2}\right) \text { with different } \\
\text { algorithms of prediction }\end{array}$ \\
\hline Model 1 & $\begin{array}{l}\text { - Asymptote estimated using a logistic } \\
\text { function } \\
\text { - Asymptote estimated using a four- } \\
\text { parameter Weibull function } \\
\text { - "Observed" minimum number of reads of } \\
\text { the } 20 \% \text { vector } \\
\text { - "Observed" maximum number of reads of } \\
\text { the } 20 \% \text { vector }\end{array}$ & $\begin{array}{l}\text { SVM }=0.9964754 \\
L M=0.9990069 \\
\text { Xboost }=0.999999\end{array}$ \\
\hline Model 2 & $\begin{array}{l}\text { - Asymptote estimated using a four- } \\
\text { parameter Weibull function } \\
\text { - "Observed" minimum number of reads of } \\
\text { the } 20 \% \text { vector } \\
\text { - "Observed" maximum number of reads of } \\
\text { the } 20 \% \text { vector }\end{array}$ & $\begin{array}{l}\text { SVM }=0.9964423 \\
L M=0.9981882 \\
\text { Xboost } 0.9999981\end{array}$ \\
\hline
\end{tabular}

TABLE 3 Model accuracy for the prediction of the maximum number of genes using only $20 \%$ of total reads in a simulation of 1,587 metatranscriptomic/ metagenomic genomic sequences 
(a)

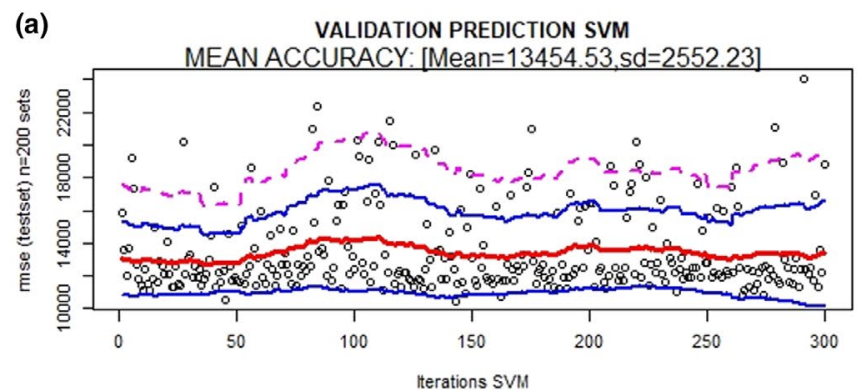

(c)

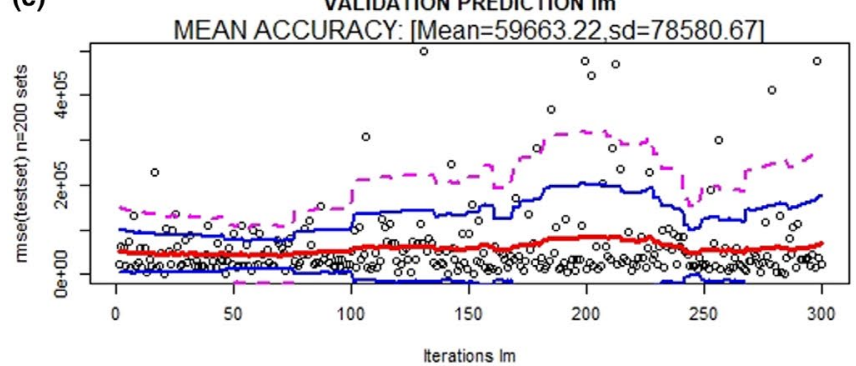

(e)

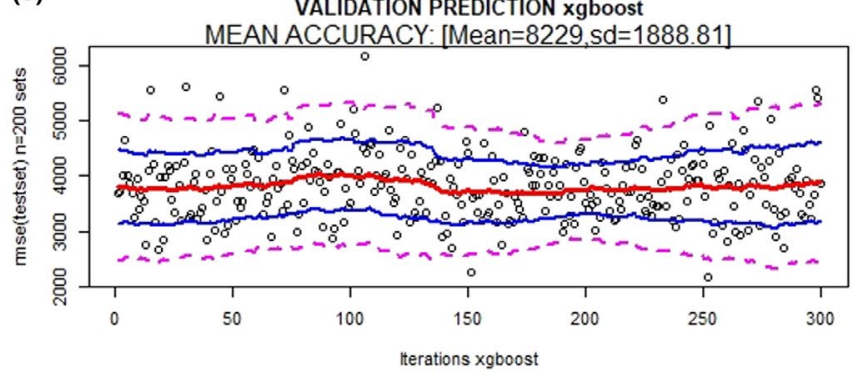

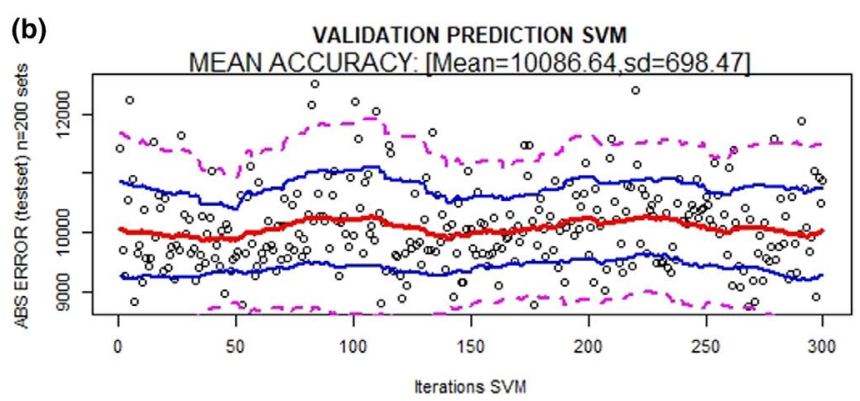

(d) VALIDATION PREDICTION Im

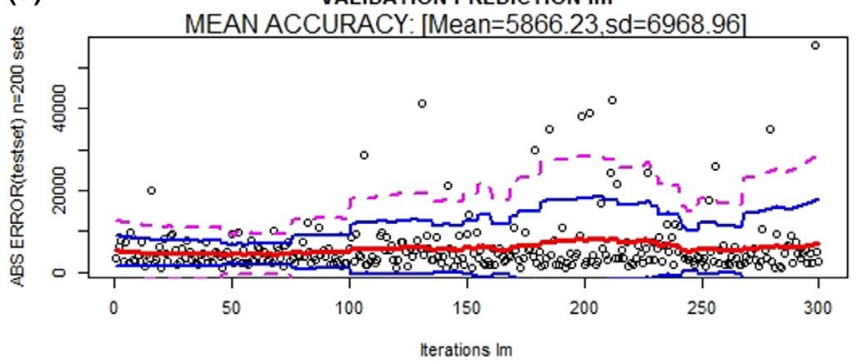

(f)

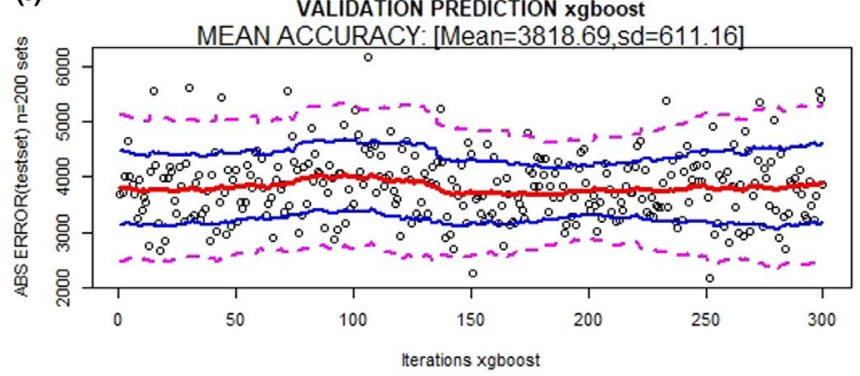

FIGURE 2 RMSE and absolute error bands (mean (red), 95\% (blue), and 99\% confidence (magenta)) of different methods [(a) support vector machine, (b) linear regression model, and (c) XBoost] using 20\% of sequencing depth (reads) to predict the maximum number of genes. 300 random resamples were performed

Using an R script (see Supplementary Material), we simulated 1,587 metatranscriptomic/metagenomic matrices containing more than $9^{9}$ reads, with random numbers of genes $(\min =267$, $\max =339,319)$ and reads $(\min =550, \max =6,823,774)$, and always 3 samples (replicas). The simulations had a high computational cost of more than 2 weeks and were carried out on a Linux Xeon SP 4114 $2.2 \mathrm{GHz}$ computer server with 40 cores. This information has been collected in a data frame for further analysis.

A rarefaction curve using the 1,587 simulated cases was computed using the function iNEXT(), and the vector obtained ( $n=100$ points, $x=$ reads, $y=$ genes) was saved and used later to compute (a) the maximum number of genes, (b) the sampling effort to reach the maximum number of genes (minimum $=1 \%$, maximum $=100 \%$; see Table 2), and c) the reads covering 90,95 and $99 \%$ of the maximum number of genes. This last part [points (a), (b), and (c) ] was done using an estimation based on the Weibull model described in Section 2.3 using nonlinear regression.

Four examples of the results obtained are shown in Figure 1. The results can be distinguished in four different types of rarefaction curves:

- Over-sampling curves: minimum sampling effort to obtain the maximum amount of genes in a quick rarefaction curve (Figure 1.a).

- Correct sampling curves: medium sampling effort to obtain the maximum amount of genes in a saturated rarefaction curve (Figure 1.b).

- Under-sampling curves: maximum sampling effort to obtain the maximum amount of genes in a nonobserved saturated rarefaction curve (Figure 1.c).

- Very under-sampling: very maximum sampling effort to obtain the maximum amount of genes in a nonobserved saturated rarefaction curve (Figure 1.d).

Moreover, in the curves of Figure 1, we can distinguish the vertical lines of the reads covering $90 \%, 95 \%$, and $99 \%$ of the maximum number of genes.

\subsection{A priori gene prediction using only a few total reads}

Using the simulated data and the parameters estimated previously, we fitted a regression to predict the potential number of genes and 
(a)

VALIDATION PREDICTION SVM

MEAN R2: [Mean $=0.996, \mathrm{sd}=0.002$ ]

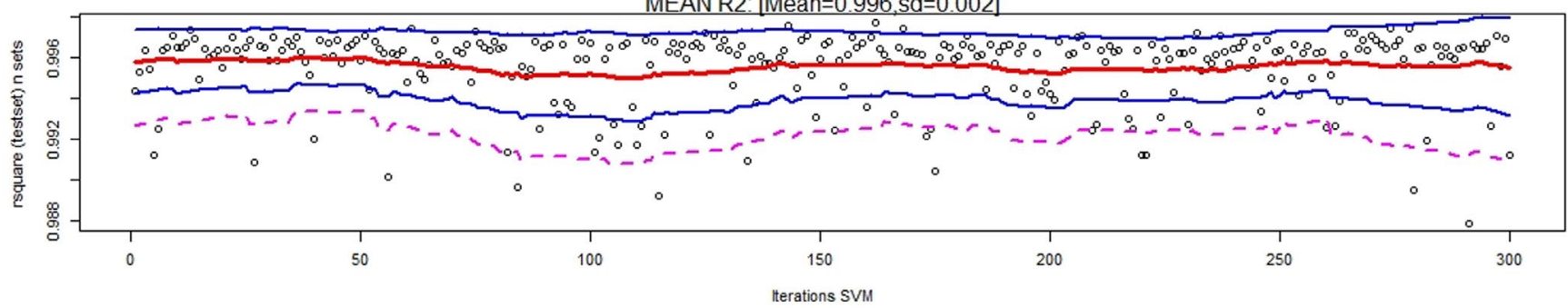

(b)

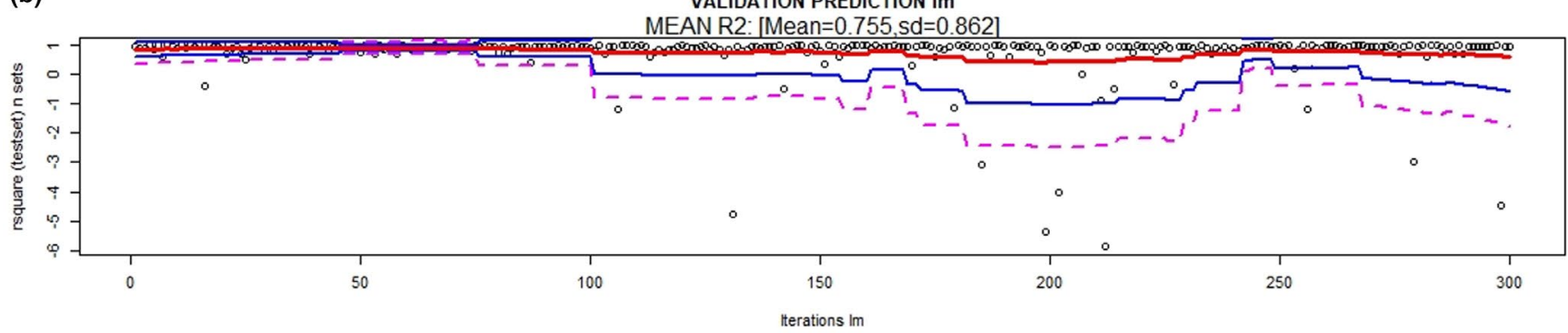

(c)

VALIDATION PREDICTION xboost

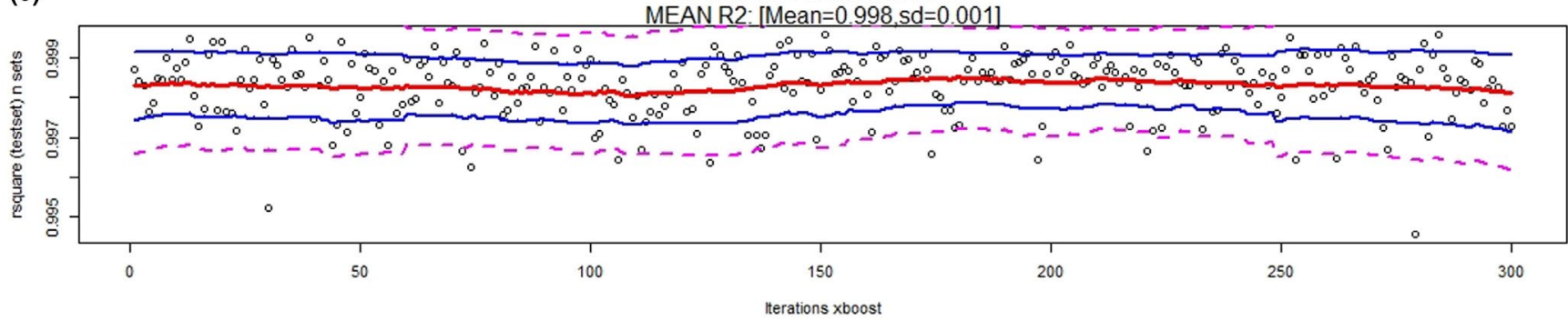

FIGURE 3 Coefficient of determination $\left(R^{2}\right.$ ) bands (mean (red), 95\% (blue) and 99\% confidence (magenta)) of the different methods used [(a) Support vector machine, (b) linear regression model, and (c) XBoost] using $20 \%$ of sequencing depth (reads) to predict the maximum number of genes. 300 random resamples were performed

the reads covering $90 \%, 95 \%$, or $99 \%$ of the maximum number of genes using only the first $20 \%$ of sequences (reads). To implement this method, we used three algorithms (linear model (Im), Extreme Gradient Boosting (XB), and support vector machine (SVM)) to predict the aforementioned values. Several predictors were tested to predict the maximum number of genes as a function of the first $20 \%$ of sequences (reads). Using the simulated data, several good predictors were detected, such as the asymptote, using a four-parameter Weibull model or other similar and well-known models such as the logistics curve model (Mendez et al., 2017). Other predictors used were the minimum-maximum number of genes observed and, finally, the minimum-maximum number of reads observed (see Table 3, central column; model 1 and model 2 and supplementary material).

After testing the prediction of the proposed models using the three aforementioned prediction algorithms (Im, Xboost and SVM), it was found that the results of the prediction of interest (maximum number of genes and reads covering $90 \%, 95 \%$, or $99 \%$ of the maximum number of genes) for the total curve with the 1,587 simulated samples were very similar, with an $R^{2}>0.99$, which indicates a possible over-fitting (see Table 3, right column).

To validate the method and the models, we initially used only the first 20 points of the rarefaction curve (reads of $20 \%$ of the total amount of the curve obtained) and then divided the total number of simulated rarefaction curves $(n=1,587)$ and the estimated parameters (maximum number of genes, sampling effort, etc.) into two parts using cross-validation: (a) In the training set, $70 \%$ was used to train and estimate the prediction models (Im, XB, and SVM), and (b) in the test set, $30 \%$ was used to check the model fit and capacity to predict the maximum number of genes, and reads covering $90 \%, 95 \%$, and $99 \%$ of the maximum number of genes using only the first $20 \%$ of sequences (reads).

We used 300 random resamplings, and a significant computational effort was made to obtain the predictions using models 1 and 2. We determined that the XB and Im are useful methods to predict the maximum number of genes using only $20 \%$ of sequencing depth. To prove the accuracy of the method, we used the mean absolute error (MAE), root-square-mean error (RSME), and the coefficient of determination $\left(R^{2}\right)$ between estimations using the Weibull model with $100 \%$ and $20 \%$ of the rarefaction curve.

The results of the validations of the three prediction methods (XB, Im, and SVM) and model 1 are presented in Figures 2 and 3 (prediction of maximum number of genes) and 4 and 5 (prediction of reads covering $95 \%$ of the maximum number of genes), which show the absolute error (MAE), RMSE bands (mean and $95 \%$ and $99 \%$ confidence), and $R^{2}$ for the 300 random resampling test sets. It can be 

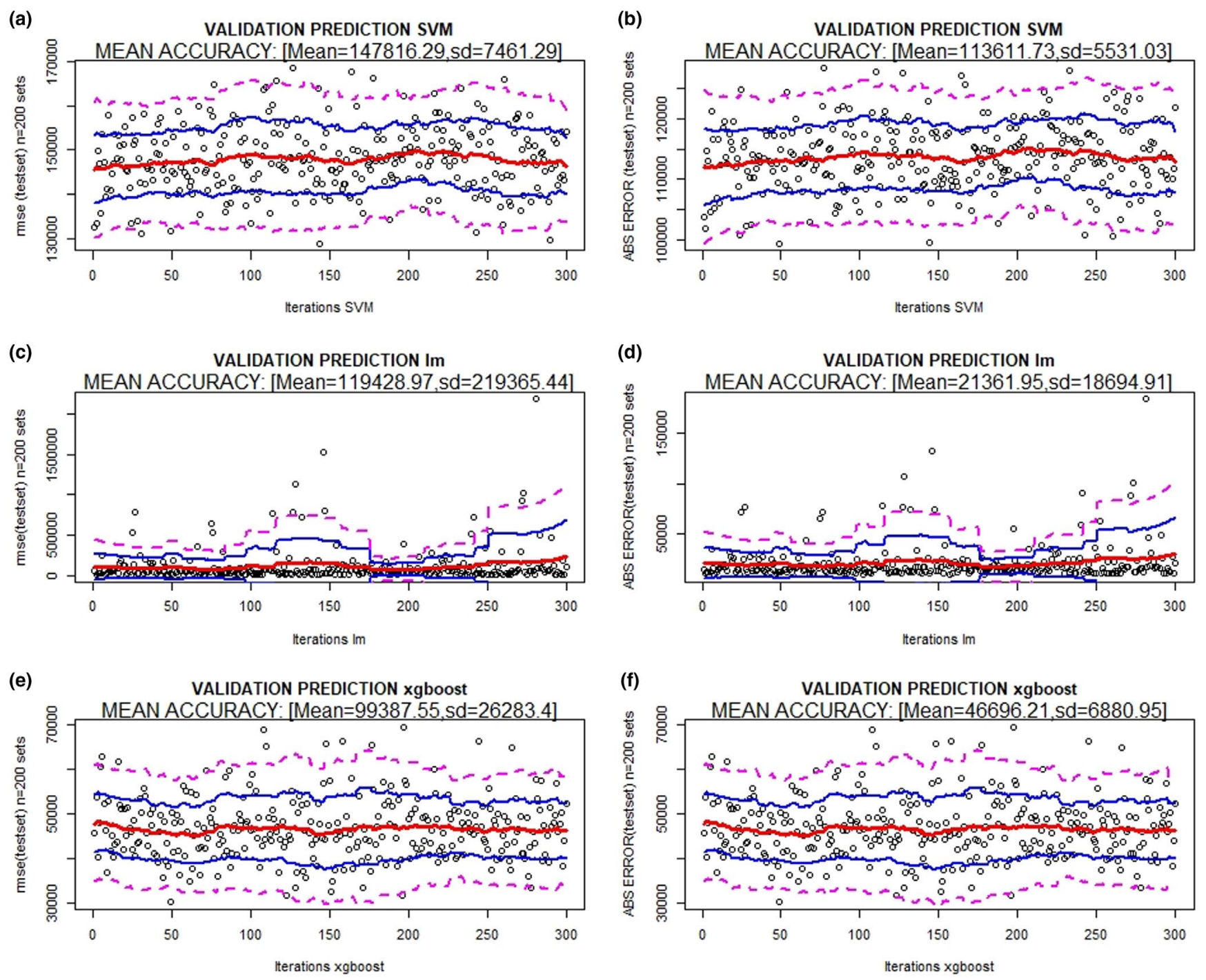

FIGURE 4 RMSE and absolute error bands (mean (red), 95\% (blue), and 99\% confidence (magenta)) of different methods [(a) Support vector machine, (b) linear regression model, and (c) XBoost] using $20 \%$ of sequencing depth (reads) to predict the reads covering $95 \%$ of the maximum number of genes. 300 random resamples were performed

observed that SVM and XB are the best methods in all situations (estimation of the maximum number of genes; number of reads to cover $95 \%$ of the maximum number of genes).

The final SVM method (model 1) for predicting the maximum number of genes has an RMSE $=13,454$, MAE $=10,086$ and $R^{2}=0.996$ between the observed and predicted values (Figure 2(a,b) and Figure 3(a). The final SVM model (model 1 ) for predicting reads to cover $95 \%$ of the maximum number of genes has an RMSE $=147,816$, $\mathrm{MAE}=113,611$ and $R^{2}=0.997$ between the observed and predicted values (Figure 4(a,b) and Figure 5(b).

The final XB model estimated to predict the maximum number of genes has an $\mathrm{RMSE}=8,229, \mathrm{MAE}=3,819$ and $R^{2}=0.998$ between the observed and predicted values (Figure 2(e,f) and Figure 3(c). The final XB model estimated to predict reads covering $95 \%$ of the maximum number of genes has an RMSE $=99,388, \mathrm{MAE}=46,696$ and $R^{2}=0.999$ between the observed and predicted values (Figure 4(e,f) and Figure 5(c).
Finally, an XB model 1 including the total amount of simulated data ( $n=1,587$ ) was estimated and saved. The $R^{2}$ of all data and prediction models (Im, XB, and SVM) are presented in Figure 6. This model will be used to predict the described parameters of interest (maximum number of genes: Figure 6 a,b,c, reads to cover $95 \%$ of the maximum number of genes: Figure $6 \mathrm{~d}, \mathrm{e}, \mathrm{f}$, effort, etc.). Also, the confidence interval (95\%) of the prediction was obtained by applying a "bagging" method, which was possible with the XB model and involves creating the same model many times (with randomness).

Finally, using 100 subsamples we obtained the prediction mean and $95 \%$ by means of the function ci.mean( ) of the library(Publish) for R. The final XB model estimated to predict the maximum number of genes has an MAE $=86$ and $R^{2}=0.9999997$ between the observed and predicted values (Figure 6c). The final XB model estimated to predict reads covering $95 \%$ of the maximum number of genes has an $\mathrm{MAE}=634$ and $R^{2}=0.9999999$ between the observed and predicted values (Figure $6 \mathrm{~g}$ ). 


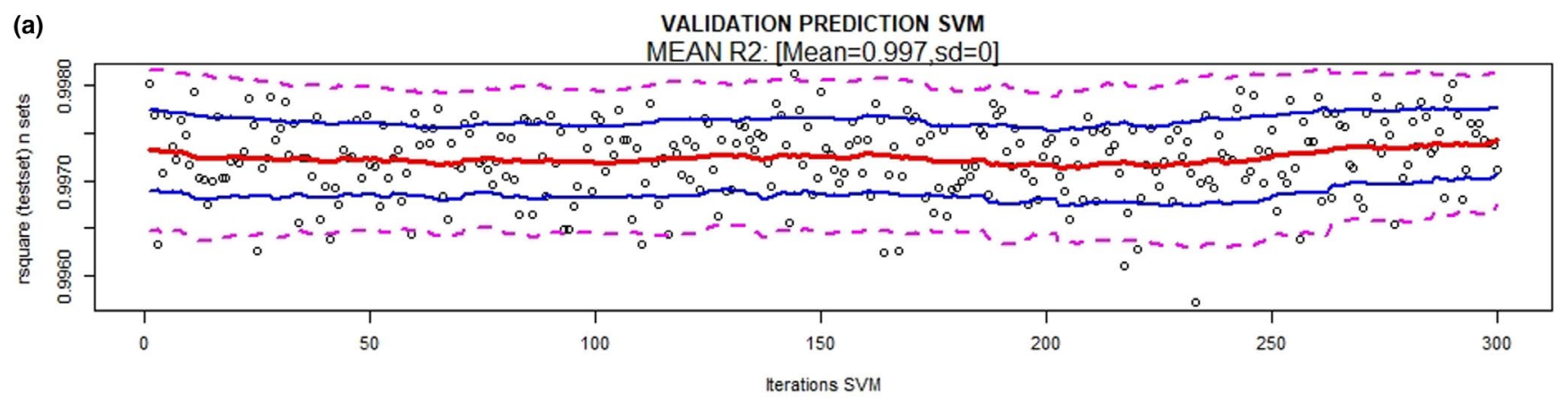

(b)

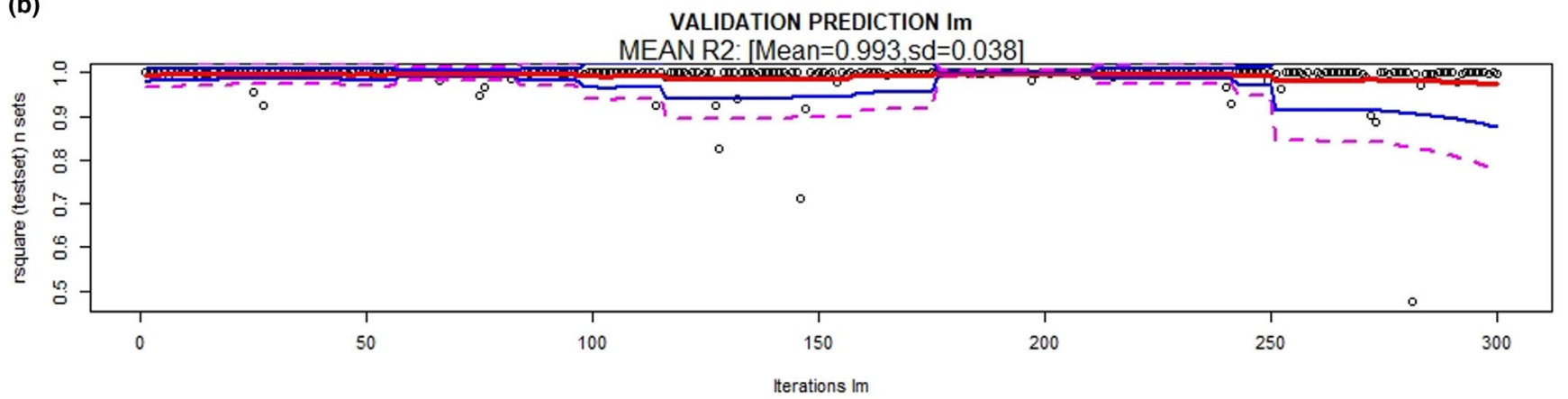

(c)

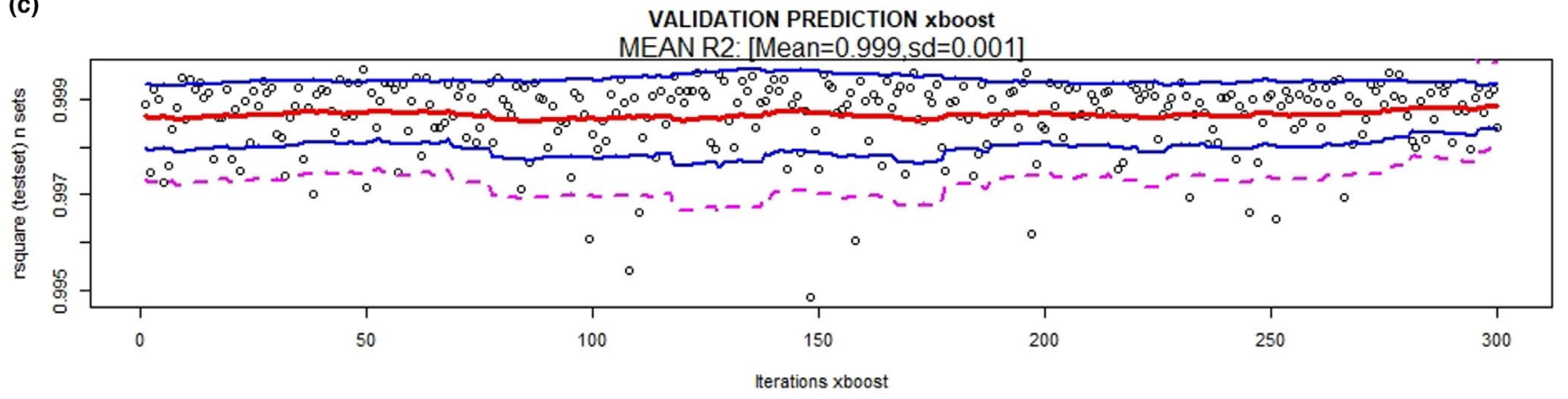

FIGURE 5 Coefficient of determination $\left(R^{2}\right)$ bands (mean (red), 95\% (blue), and 99\% confidence (magenta)) of the different methods used. [(a) Support vector machine, (b) linear regression model, and (c) XBoost] using $20 \%$ of sequencing depth (reads) to predict the reads covering $95 \%$ of the maximum number of genes. 300 random resamples were performed

\subsection{Application of the proposed method to real data}

External validation (real data not used before) was performed to check the algorithms developed previously. To this end, we used a set of 15 datasets of metatranscriptomes from the oral cavity. These RNA sequences consist of vectors of $10^{5}-1.5 \times 10^{7}$ read depth with a 10,000 and 600,000 gene size, most of them with saturation but in some cases with a definite unsaturation. We used these sequences to validate the method and predict the maximum number of genes and the number of reads covering $95 \%$ of the maximum number of genes, using all the available reads or only a percentage (3\%, $20 \%$, and $60 \%$ of read depth). The function monle.predict.max( ) was developed to compute this type of incomplete transcriptomic vectors ( $X=$ sequencing depth, $Y=$ genes).

The results of this validation are shown in the supplementary material and reflect that the used model, based on a four-parameter
Weibull model, had a perfect fit and could correctly estimate the parameters of interest (maximum number of genes, read depth covering $95 \%$ of the maximum number of genes).

When only a percentage (3\%, $20 \%$, and $60 \%$ of read depth) of the transcriptomic vector was used, the results were also quite acceptable for predicting the maximum amount of genes and moderately acceptable for predicting the reads covering $95 \%$ of the maximum number of genes. The prediction for the maximum number of genes was considered acceptable when the maximum number of genes was within the XB bagging $95 \%$ prediction interval. Similarly, the prediction of the read depth covering $95 \%$ of the maximum number of genes was considered acceptable when within the XB bagging 95\% prediction interval or between the $90 \%-99 \%$ interval calculated using the $100 \%$ read depth of the transcriptome.

When $3 \%\left(10^{5}-5 \times 10^{5}\right.$ reads) was used to predict the parameters of interest, $12 / 15$ (80\%) curves to predict the number of genes and $6 / 15$ (33\%) curves to predict the reads covering $95 \%$ 
(a)

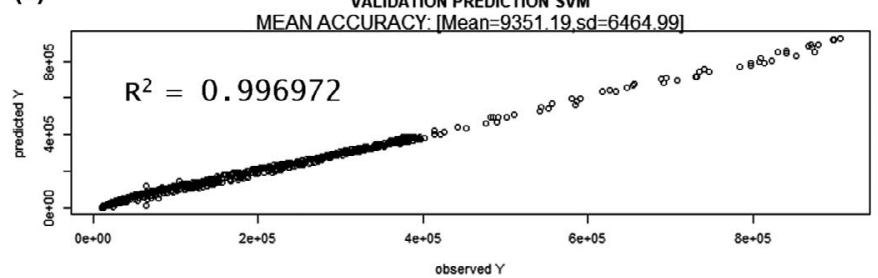

(b)

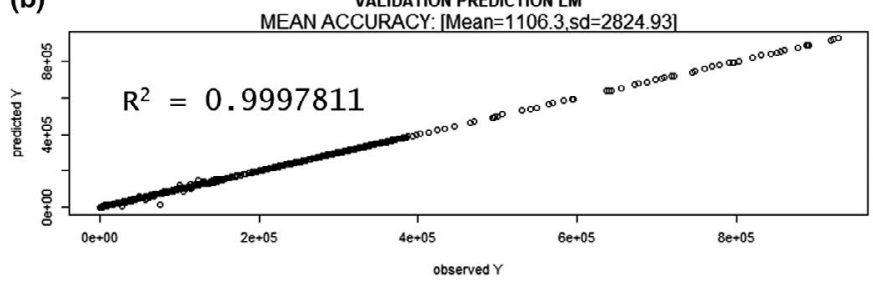

(c)

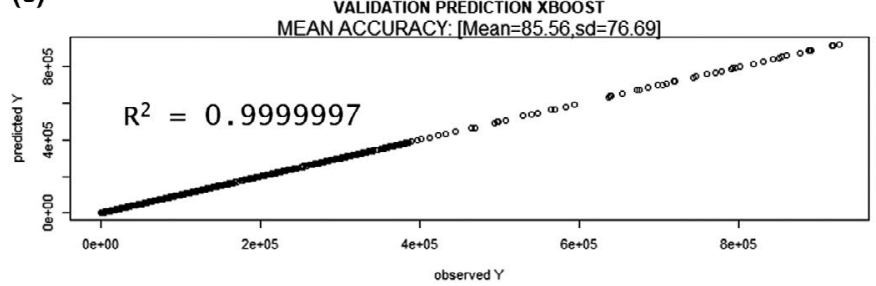

(e)

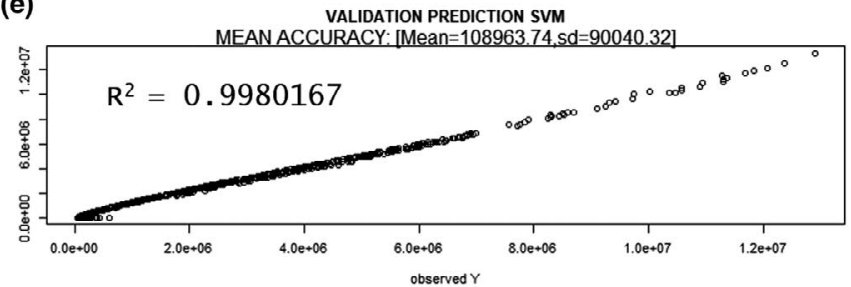

(f)

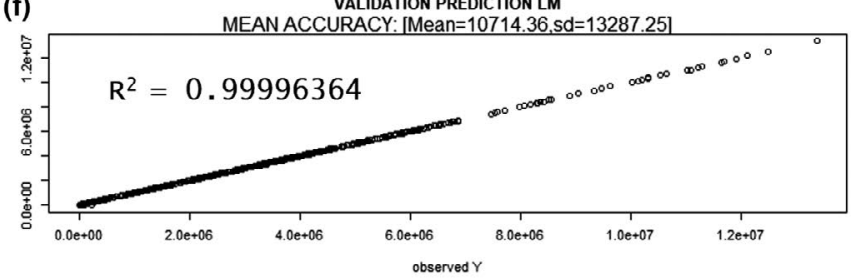

(g)

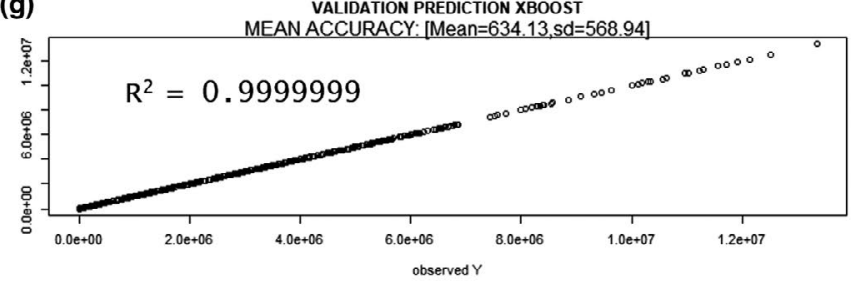

FIGURE 6 Prediction of maximum number of genes (a, b, c) and reads covering $95 \%$ of the maximum number of genes (d, e, $f$ ) using a SVM, Im, and XGBoost model and only $20 \%$ of the reads versus the observed value. All the samples were used $(n=1,556)$

of the maximum number of genes were acceptable. When $20 \%$ (105-3 $\times 106$ reads) was used to predict the parameters of interest, 14/15 (93\%) curves to predict the number of genes and $9 / 15(60 \%)$ curves to predict reads covering $95 \%$ of the maximum number of genes were acceptable. When $60 \%$ (105-1 $\times 107$ reads) were used to predict the parameters of interest, 14/15 (90\%) curves to predict the number of genes and 9/15 (60\%) curves to predict reads covering $95 \%$ of the maximum number of genes were acceptable.

\section{4 | Conclusions}

This proposed method to estimate the maximum number of genes and the reads covering 90,95 , and $99 \%$ of the maximum number of genes, using an algorithm based on a rarefaction curve + Weibull model + machine learning prediction, will help researchers to know whether sampling is sufficient or needs to be increased. The method should be used with precaution when predicting the sequencing depth, especially with unsaturated samples. However, although the proposed model can cause predictive problems, it was found to work in most cases. Further studies using real sequences and typologies should be carried out to fully validate the model and the simulation-based methodology.

Estimating the sequencing depth required to adequately sample the target metatranscriptome/metagenome using RNA-seq, and Shotgun is an essential first step in obtaining robust results in subsequent analysis and avoiding overexpansion once the information contained in the library reaches saturation. Our method allows the use of an initial shallowly sequenced sample (in this case $20 \%$ of the total amount of reads sampled) to estimate the sequencing effort needed to cover the whole metatranscriptome/ metagenome from the same sample and therefore to estimate the sample size. The initial number of sequences is low enough for current NGS methods to analyze a considerable number of samples at a low cost.

\section{ACKNOWLEDGMENT}

Our thanks to Andreu Paytuví and Walter Sanseverino from Sequentia-Biotech for his advice with metagenomics. Also, our thanks to Javier Mendez from Department of Microbiology of the University of Barcelona for his advice with the methods.

\section{CONFLICT OF INTEREST}

The authors declare no conflict of interest.

\section{AUTHOR CONTRIBUTIONS}

Toni Monleon-Getino: Conceptualization (equal); data curation (lead); formal analysis (lead); investigation (equal); methodology (equal); software (equal); writing - original draft (equal); writing review and editing (equal). Jorge Frias-Lopez: Conceptualization (equal); methodology (equal); writing - original draft (lead); writing - review and editing (lead).

\section{DATA AVAILABILITY STATEMENT}

All code is open source and available in Github. All functionalities shown in Figure 1 (rarefaction curve, Weibull nonlinear model, 
effort estimation, extrapolation of the maximum number of genes, reads covering 90,95 , and $99 \%$ of the maximum number of genes) have been compiled in two new functions in R: PILI3( ) and monle. predict.max ( ) and added to the library(Sequencingeffort) and can be found at the repository https://github.com/amonleong/Seque ncingeffort. For each described function, at least two examples of use are given, as well as the explanation of the arguments of the functions.

\section{ORCID}

Toni Monleon-Getino (iD https://orcid.org/0000-0001-8214-3205 Jorge Frias-Lopez iD https://orcid.org/0000-0002-2097-3171

\section{REFERENCES}

Belda-Ferre, P., Alcaraz, L. D., Cabrera-Rubio, R., Romero, H., SimónSoro, A., Pignatelli, M., \& Mira, A. (2012). The oral metagenome in health and disease. ISME Journal, 6, 46-56. https://doi.org/10.1038/ ismej.2011.85

Benítez-Páez, A., Belda-Ferre, P., Simón-Soro, A., \& Mira, A. (2014). Microbiota diversity and gene expression dynamics in human oral biofilms. BMC Genomics., 15, 311. https://doi. org/10.1186/1471-2164-15-311

Chang, C.-C., \& Lin, C.-J. (2011). A library for support vector machines. ACM Transactions on Intelligent Systems and Technology, 2(27), 1-27. https://doi.org/10.1145/1961189.1961199

Chen, T., \& Guestrin, C. (2016). XGBoost: A Scalable Tree Boosting System. Proceedings of the 22nd ACM SIGKDD International Conference on Knowledge Discovery and Data Mining, San Francisco, California USA; 785-794. https://www.kdd.org/kdd2016/ papers/files/rfp0697-chenAemb.pdf

Cortes, C., \& Vapnik, V. (1995). Support-vector networks. Machine Learning, 20, 273-297. https://doi.org/10.1007/BF00994018

CRAN (2018a). LearnBayes: Functions for Learning Bayesian Inference version 2.15.1 from [Internet]. Retrieved from https://rdrr.io/cran/Learn Bayes/

CRAN (2018b). nls2: Non-linear regression with brute force version 0.2 from CRAN [Internet]. Retrieved from https://rdrr.io/cran/nls2/

Dewhirst, F. E., Chen, T., Izard, J., Paster, B. J., Tanner, A. C. R., Yu, W.-H., Lakshmanan, A., \& Wade, W. G. (2010). The human oral microbiome. Journal of Bacteriology, 192, 5002-5017. https://doi.org/10.1128/ JB.00542-10

García-Ortega, L. F., \& Martínez, O. (2015). How many genes are expressed in a transcriptome? Estimation and results for RNA-Seq. PLoS One, 10, e0130262. https://doi.org/10.1371/journal.pone.0130262

Haas, B. J., Chin, M., Nusbaum, C., Birren, B. W., \& Livny, J. (2012). How deep is deep enough for RNA-Seq profiling of bacterial transcriptomes? BMC Genomics, 13, 734. https://doi. org/10.1186/1471-2164-13-734

Haffajee, A. D., Socransky, S. S., Patel, M. R., \& Song, X. (2008). Microbial complexes in supragingival plaque. Oral Microbiology and Immunology, 23, 196-205. https://doi.org/10.1111/j.1399-302X.2007.00411.x

Hsieh, T. C., Ma, K. H., \& Chao, A. (2016). iNEXT: An R package for rarefaction and extrapolation of species diversity (Hill numbers). Methods in Ecology and Evolution, 7, 1451-1456.

Hyndman, R. J., \& Koehler, A. B. (2006). Another look at measures of forecast accuracy. International Journal of Forecasting, 22, 679-688. https://doi.org/10.1016/j.ijforecast.2006.03.001

Jorth, P., Turner, K. H., Gumus, P., Nizam, N., Buduneli, N., \& Whiteley, M. (2014). Metatranscriptomics of the human oral microbiome during health and disease. MBio, 5, e01012-e01014. https://doi. org/10.1128/mBio.01012-14
Kolenbrander, P. E. (2000). Oral microbial communities: Biofilms, interactions, and genetic systems. Annual Review of Microbiology, 54, 413-437. https://doi.org/10.1146/annurev.micro.54.1.413

Kolenbrander, P. E., Andersen, R. N., Blehert, D. S., Egland, P. G., Foster, J. S., \& Palmer, R. J. J. (2002). Communication among oral bacteria. Microbiology and Molecular Biology Reviews: MMBR, 66, 486-505. https://doi.org/10.1128/MMBR.66.3.486-505.2002

Maindonald, J. H., \& Braun, W. J. (2010). Data analysis and graphics using $R$-an Example-Based Approach (3rded.). Cambidge, UK:Cambridge Series in Statistical and Probabilistic Mathematics.

Maindonald, J. H., \& Braun, W. J. (2019). Data analysis and graphics data and functions (DAAG). Retrieved from https://cran.r-project.org/web/ packages/DAAG/DAAG.pdf

Marsh, P. D. (2006). Dental plaque as a biofilm and a microbial community - Implications for health and disease. BMC Oral Health, 6(S1), 1-7. https://doi.org/10.1186/1472-6831-6-S1-S14

Mendez, J., Monleon-Getino, A., Jofre, J., \& Lucena, F. (2017). Use of non-linear mixed-effects modelling and regression analysis to predict the number of somatic coliphages by plaque enumeration after 3 hours of incubation. J Water Health, 15, 706-717.

Monleon-Getino, A., Rodriguez-Casado, C. I., \& Mendez-Viera, J. (2017). Sample size in metagenomics, a Bayesian approach using BDSbiost3 for R. Proc CEB 2017. Sevilla, Spain.

Monleon-Getino, T., Rodríguez-Casado, C. I., \& Verde, P. E. (2019). The Shannon entropy ratio: A Bayesian biodiversity index applied to the measure of uncertainty in metagenomic communities (putative enterotypes). Journal of Advanced Statistics, 4(4), 23-34.

Ni, J., Yan, Q., \& Yu, Y. (2013). How much metagenomic sequencing is enough to achieve a given goal? Scientific Reports, 3, 1-7.

Paster, B. J., Boches, S. K., Galvin, J. L., Ericson, R. E., Lau, C. N., Levanos, V. A., Sahasrabudhe, A., \& Dewhirst, F. E. (2001). Bacterial diversity in human subgingival plaque. Journal of Bacteriology, 183, 3770-3783.

Peterson, S. N., Snesrud, E., Liu, J., Ong, A. C., Kilian, M., Schork, N. J., $\&$ Bretz, W. (2013). The dental plaque microbiome in health and disease. PLoS One, 8, e58487.

Pinheiro, J. (2018). Mixed-effects models in S and S-PLUS. Springer. Retrieved from https://www.springer.com/us/book/9780387989570

Mangiafico, S. S. (2015). An R Companion for the Handbook of Biological Statistics [Internet]. Retrieved from https://rcompanion.org/rcomp anion/a_02.html

Robinson, D. G., \& Storey, J. D. (2014). subSeq: Determining appropriate sequencing depth through efficient read subsampling. Bioinformatics, 30, 3424-3426.

Rodríguez-Casado, M.-G., Cubedo, M., \& Ríos-Alcolea, M. (2017). A priori groups based on Bhattacharyya distance and partitioning around medoids algorithm (PAM) with applications to metagenomics. IOSR Journal of Mathematics, 13(3), 24-32.

Shakya, M., Lo, C. C., \& Chain, P. S. G. (2019). Advances and challenges in metatranscriptomic analysis. Frontiers in Genetics, 10, 904. https:// doi.org/10.3389/fgene.2019.00904

Socransky, S. S., Haffajee, A. D., Cugini, M. A., Smith, C., \& Kent, R. L. J. (1998). Microbial complexes in subgingival plaque. Journal of Clinical Periodontology, 25, 134-144. https://doi.org/10.1111/j.1600051X.1998.tb02419.x

Tamames, J., de la Peña, S., \& de Lorenzo, V. (2012). COVER: A priori estimation of coverage for metagenomic sequencing. Environmental Microbiology Reports, 4,335-341. https://doi.org/10.1111/j.1758-2229.2012.00338.x

Toung, J. M., Morley, M., Li, M., \& Cheung, V. G. (2011). RNA-sequence analysis of human B-cells. Genome Research, 21, 991-998. https:// doi.org/10.1101/gr.116335.110

Westermann, A. J., Gorski, S. A., \& Vogel, J. (2012). Dual RNA-seq of pathogen and host. Nature Reviews Microbiology, 10, 618-630. https://doi.org/10.1038/nrmicro2852

Yost, S., Duran-Pinedo, A. E., Teles, R., Krishnan, K., \& Frias-Lopez, J. (2015). Functional signatures of oral dysbiosis during periodontitis 
progression revealed by microbial metatranscriptome analysis. Genome Medicine, 7, 27. https://doi.org/10.1186/s13073-015-0153-3

Zhang, X., Li, L., Butcher, J., Stintzi, A., \& Figeys, D. (2019). Advancing functional and translational microbiome research using meta-omics approaches. Microbiome, 7(1), 154. https://doi.org/10.1186/s4016 8-019-0767-6

\section{SUPPORTING INFORMATION}

Additional supporting information may be found online in the Supporting Information section.
How to cite this article: Monleon-Getino T, Frias-Lopez J. A priori estimation of sequencing effort in complex microbial metatranscriptomes. Ecol Evol. 2020;00:1-13. https://doi. org/10.1002/ece3.6941 